\title{
An evaluation of the effectiveness of the Eatwell for Life programme in Nottingham
}

\author{
J. Woods ${ }^{1}$ and A. McCamley ${ }^{2}$ \\ ${ }^{1}$ Nottingham Community Nutrition and Dietetic Service, Nottingham CityCare Partnership, Aspect House, \\ Bennerley Road, Nottingham, NG6 8WR and ${ }^{2}$ Faculty of Development and Society, Sheffield Hallam University, \\ Collegiate Campus, Sheffield, S10 2BP
}

The Eatwell for Life (EWL) programme is a 6 week community-based dietary intervention which aims to increase nutritional knowledge, cooking confidence and skills to support behaviour change in relation to eating a balanced diet. Registered Nutritionists and Dietitians developed the programme and have trained and support a team of Community Food Workers (CFW) who facilitate the courses. CFW lead on practical cooking activities, nutrition education and utilise behaviour change techniques such as goal setting, to help participants make practical changes to their eating habits. Nutrition and cooking skills interventions are a popular way to improve dietary intake, cooking skills and health related outcomes ${ }^{(1)}$ however, there is a need for studies to evaluate the long-term effectiveness $^{(2)}$. Many have used brief measures but few extend their consideration of impact past the end of the course and into the follow up months. The aim of the study was to evaluate the effectiveness of EWL, with a particular focus on longer-term effectiveness in terms of dietary behaviour and the wider impact.

A mixed method evaluation was conducted. A self-reported questionnaire was administered pre intervention $(\mathrm{T} 1, \mathrm{n}=69)$ and post intervention $(\mathrm{T} 2, \mathrm{n}=66$ ), which measured consumption of fat, sugar, fruit and vegetables, confidence in cooking and preparing healthy food and dietary change made by the individual, family and friends. Fruit and vegetable consumption was also measured at 6 months follow up (T3, $n=22)$. Qualitative data on the long-tern effectiveness of EWL was collected via focus groups and semistructured telephone interviews, which were conducted at 3 and 6 months follow up with a purposive sample of EWL participants.

Statistically significant increases between $\mathrm{T} 1$ and $\mathrm{T} 2$ in average daily fruit $(0.85$ portions, $\mathrm{P}<0.001)$ and vegetable $(0.99$ portions, $\mathrm{P}<0.001)$ intake were found. Paired means were analysed in a sub sample of 22 participants who responded to a follow up postal questionnaire between $\mathrm{T} 1$ to $\mathrm{T} 2$ and $\mathrm{T} 2$ to $\mathrm{T} 3$. Vegetable consumption between $\mathrm{T} 1$ and T3significantly increased (1.36 portions, $\mathrm{P}<0.001$ ). A reduction in median values of sugar consumption was reported between T1 (once a day) and T2 (once a week or less).

A further sample $(n=22)$ of EWL participants was involved in the qualitative methods. Key themes emerged from a thematic analysis of the qualitative data. These themes indicated that participants were now cooking from scratch and reducing the amount of convenience foods consumed; knowledge of key healthy eating messages had increased; dietary changes made in terms of eating, cooking and shopping habits; wider impact in terms of influencing family and friends diets and gaining a social element from the course.

The EWL programme led to positive and sustained changes in participants cooking and eating behaviours. This study adds to the existing limited evidence base and shows promise as a way to improve dietary behaviours over the longer term contributing to obesity prevention and reducing health inequalities. The wider impact of EWL has potential to show a positive social return on investment demonstrating the full impact of the programme.

1. Garcia AL, Vargas E, Lam PS et al. (2013). Public Health Nutr 17, 1013-1021.

2. Reicks M, Trofholz AC, Jamie S et al. (2014). J of Nutr Ed and Beh 46, 259-276. 